\title{
Stellar population properties in the nuclei and bulges of nearby lenticular galaxies
}

\author{
Olga K. Sil'chenko ${ }^{1}$ \\ ${ }^{1}$ Sternberg Astronomical Institute, Moscow State University, University av. 13, Moscow \\ 119992, Russia email: olga@sai.msu.su
}

\begin{abstract}
The properties of stellar populations in the centers of nearby lenticular galaxies are investigated by means of $2 \mathrm{D}$ spectroscopy. All the galaxies are divided into 4 groups depending on the environment type; every subsample contains more than 10 galaxies. Clear distinctions between the mean stellar ages and abundance ratios are found both for the nuclei and for the bulges of the S0s in the different environments.
\end{abstract}

During 1996-2003 we have obtained 2D spectroscopic data in the spectral range of 4600-5400 $\AA$ for 54 nearby lenticular galaxies over various types of environments, namely, for the field galaxies, for the brightest group galaxies ('group centers') and secondary group members, and for the cluster lenticular galaxies. The observations have been made with the Multi-Pupil Spectrograph of the $6 \mathrm{~m}$ telescope (MPFS). Here we compare the stellar population properties in the nuclei and in the 'bulges' of these galaxies, the term 'bulges' being ascribed to the rings between $R=3^{\prime \prime}$ and $R=7^{\prime \prime}$. The stellar population abundances and ages are determined by comparing measured Lick indices Mgb, Fe5270, Fe5335, and $\mathrm{H} \beta$ to the evolutionary synthesis models of Thomas, Maraston, \& Bender (2003). As we have reported earlier (Sil'chenko 1993, Sil'chenko 2002), a significant part of nearby lenticular galaxies possess young stellar nuclei; according to the present estimates, among field S0s and group second- or third-ranked members, $50 \%$ of the galaxies, and among group centers and cluster members, $25 \%$ of the galaxies reveal a mean age of the nuclear stellar population of less than 5 Gyr. A bulge is almost always older than the nucleus. An effect of environments is seen both for the nuclei and for the bulges: if we consider the clusters (Virgo and Ursa Major) and group centers as dense environments and the field and group periphery as sparse environments, then in the dense environments the stellar populations of S0s are in average older by 4-5 Gyr than in the sparse ones.

We have analysed carefully the index-index diagrams, $\langle\mathrm{Fe}\rangle \equiv(\mathrm{Fe} 5270+\mathrm{Fe} 5335) / 2$ vs $\mathrm{Mgb}$ and $\mathrm{H} \beta$ vs $[\mathrm{MgFe}] \equiv \sqrt{\mathrm{Mgb}\langle\mathrm{Fe}\rangle}$, for all four types of environments; the stellar absorption-line index $\mathrm{H} \beta$ has been corrected for the emission contamination mostly by using the $\mathrm{H} \alpha$ emission estimates from Ho et al. (1997). We have come to the following particular conclusions:

(a) In the dense environments, $[\mathrm{Mg} / \mathrm{Fe}]_{n u c}$ varies from +0.2 to +0.4 under $[\mathrm{m} / \mathrm{H}] \approx$ const $\approx+0.3$. This result implies very brief and very effective last star formation epochs in the nuclei of the lenticulars in the dense environments.

(b) In the sparse environments, $[\mathrm{Mg} / \mathrm{Fe}]_{n u c} \approx$ const $\approx+0.1-+0.2$, while $[\mathrm{m} / \mathrm{H}]$ varies from -0.3 to +0.3 . The metallicity variations seem to reflect a well-known massmetallicity relation for early-type galaxies.

(c) The bulges within each type of environments represent a metallicity sequence under $[\mathrm{Mg} / \mathrm{Fe}] \approx$ const, exactly as the nuclei of the lenticulars in the sparse environments, but the mean $[\mathrm{Mg} / \mathrm{Fe}]$ falls when passing from the dense environments to the sparse ones. 

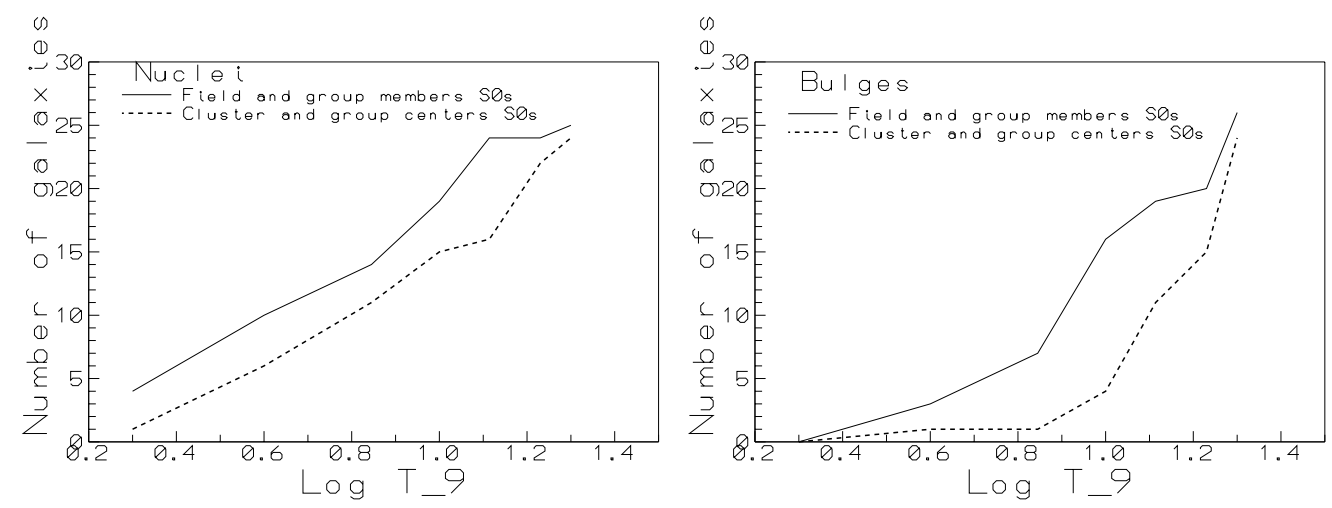

Figure 1. Cumulative age distributions: a number of objects younger than abcissa which is $\lg T$ in Gyr. (a) The stellar nuclei of the galaxies $(b)$ The bulges taken in the rings between $R=3^{\prime \prime}$ and $R=7^{\prime \prime}$.

(d) Among the nuclei of S0s of all types of environments one can find mean stellar ages from 1 Gyr to 15 Gyr.

(e) The bulges have mean stellar ages between 5 and 15 Gyr, the bulges in the field lenticulars being significantly younger than in S0s of other types of environments. However, we must note that in some galaxies what we regard as the 'bulges' may be in fact a sum of the bulges and of extended circumnuclear disks produced by secondary star formation bursts. If a circumnuclear disk is close to face-one orientation, it cannot be detected either by photometry nor by rotation.

(f) Cumulative age distributions (Fig. 1) reveal a systematic shift between the stellar age distributions of both the nuclei and the bulges in the dense and the sparse environments: the median ages of the nuclei are $8 \mathrm{Gyr}$ in the dense environments and $5.5 \mathrm{Gyr}$ in the sparse ones, and the median ages of the bulges are 14 Gyr and 9 Gyr, respectively.

These observational results must be compared with the predictions of the cosmologybased scenario of galaxy formation which imply environment dependence of the formation epoch similar to that found by us in this work. However I feel that the absolute mean age values for the nuclei and bulges of the nearby luminous S0 galaxies may be in contradiction with the prescriptions of the $\Lambda \mathrm{CDM}$ model. I would stress also the difference between the nuclei and the bulges which appear to be quite different stellar subsystems, with absolutely decoupled evolutions. The secondary star formation bursts are obviously more frequent in the nuclei than over extended areas in the bulges.

\section{Acknowledgements}

I thank my collaborators V. L. Afanasiev, A. N. Burenkov, A. V. Moiseev, and V. V. Vlasyuk. The study of young nuclei in lenticular galaxies has been supported by the Russian Foundation for Basic Research (grant 01-02-16167) and by the Federal ScientificTechnical Program - contract of the Science Ministry of Russia no.40.022.1.1.1101.

\section{References}

Ho, L. C., Filippenko, A. V., \& Sargent, W. L. W. 1997, ApJS, 112, 315

Sil'chenko, O. K. 1993, Pis'ma v AZh, 19, 693

Sil'chenko, O. K. 2002, in Galaxies: the Third Dimension (eds. Rosado M., Binette L., and Arias L.) ASP Conf. Proc., 282, 121

Thomas D., Maraston C., \& Bender R. 2003, MNRAS, 339, 897 